\title{
In vitro tissue engineering of a cardiac graft using a degradable scaffold with an extracellular matrix-like topography
}

\author{
Osamu Ishii, MD, ${ }^{\mathrm{a}, \mathrm{b}}$ Michael Shin, PhD, ${ }^{\mathrm{a}}$ Taijiro Sueda, MD, PhD, ${ }^{\mathrm{b}}$ and Joseph P. Vacanti, MD
}
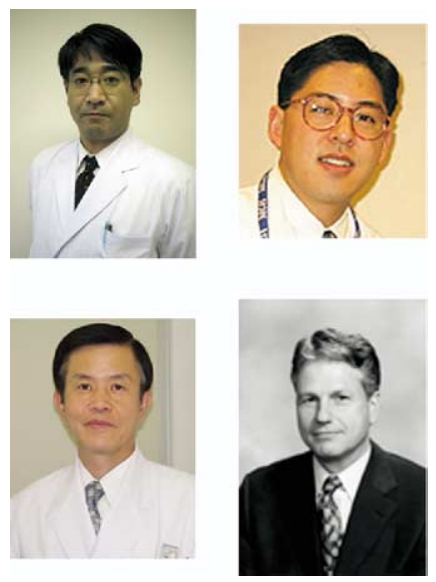

Top, Drs Ishii and Shin Bottom, Drs Sueda and Vacanti

From the Department of Surgery, Massa-
chusetts General Hospital and Harvard
Medical School, ${ }^{\text {a }}$ Boston, Mass, and the
First Department of Surgery, Hiroshima
University School of Medicine, ${ }^{\text {b Hiro- }}$
shima, Japan.

Supported by a grant from the Center for the Integration of Medicine and Innovative Technology (CIMIT).

Received for publication Feb 8, 2005; revisions received May 9, 2005; accepted for publication May 18, 2005.

Address for reprints: Michael Shin, PhD, Department of Surgery, Massachusetts General Hospital and Harvard Medical School, Boston, MA 02114 (E-mail: michael.shin@gmail.com).

J Thorac Cardiovasc Surg 2005;130:1358-63

$0022-5223 / \$ 30.00$

Copyright (C) 2005 by The American Association for Thoracic Surgery

doi:10.1016/j.jtcvs.2005.05.048
Objective: Cardiac tissue engineering has been proposed as a treatment to repair impaired hearts. Bioengineered cardiac grafts are created by combining autologous cell transplantation with a degradable scaffold as a temporary extracellular matrix. Here we present a system for engineered myocardium combining cultured cardiomyocytes and a novel biodegradable scaffold with a unique extracellular matrix-like topography.

Methods: Cardiomyocytes were harvested from neonatal rats and cultured in vitro on biodegradable electrospun nanofibrous poly( $\varepsilon$-caprolactone) meshes. Between days 5 and 7, the meshes were overlaid to construct 3-dimensional cardiac grafts. On day 14 of in vitro culture, the engineered cardiac grafts were analyzed by means of histology, immunohistochemistry, and scanning electron microscopy.

Results: The cultured cardiomyocytes attached well to the meshes, and strong beating was observed throughout the experimental period. The average fiber diameter of the scaffold is about $250 \mathrm{~nm}$, well below the size of an individual cardiomyocyte. Hence the number of cell-cell contacts is maximized. Constructs with up to 5 layers could be formed without any incidence of core ischemia. The individual layers adhered intimately. Morphologic and electrical communication between the layers was established, as verified by means of histology and immunohistochemistry. Synchronized beating was also observed.

Conclusions: This report demonstrates the formation of thick cardiac grafts in vitro and the versatility of biodegradable electrospun meshes for cardiac tissue engineering. It is envisioned that cardiac grafts with clinically relevant dimensions can be created by using this approach and combining it with new technologies to induce vascularization.

$\mathrm{H}$ eart failure is one of the leading causes of morbidity and mortality in industrialized nations. Because of the limited ability of the heart to repair itself, surgical intervention is frequently required to treat patients with severe cardiac problems. Although treatments for both structural and functional problems have become highly advanced, whole organ replacement remains the only option when more conservative approaches fail. Since the 1960s, heart transplantation has become the preferred treatment for patients with chronic, irreversible heart failure, but limited donor organ supply and organ rejection remain critical problems. $^{1}$

The concept of tissue engineering was developed to alleviate the shortage of donor organs. The objective of tissue engineering is to develop laboratory-grown tissue or organs to replace or support the function of defective or injured body parts. $^{2}$ Tissue engineering is an interdisciplinary approach that relies on the synergy of cell biology, materials engineering, and reconstructive surgery to achieve its goal. One key approach in tissue engineering is the combined transplantation of cells and 
biodegradable scaffolds to provide structural support and guide tissue regeneration. The fundamental hypothesis underlying tissue engineering is that dissociated healthy cells will reorganize into functional tissue when given the proper structural support and signals. Given the appeal of this concept, it was anticipated that cardiovascular tissue engineering would become the treatment of the next generation. However, this potential has not been fulfilled yet because of several important considerations. Tissue engineering of a complete heart remains a dream of the future, but substantial progress has been made toward the development of individual components. Initial research in cardiovascular tissue engineering focused primarily on blood vessels and heart valves. ${ }^{3,4}$

Recently, attention has shifted to the myocardium, and several groups have reported encouraging results in the tissue engineering of functional heart grafts. Several groups have relied on 3-dimensional (3-D) scaffolds to create cardiac grafts. However, if the scaffold is too stiff, it can interfere with the contractile motion of the cardiomyocytes. Therefore it is imperative to choose a material with appropriate mechanical properties.

To this end, several materials have been assessed. Li and associates $^{5-7}$ produced 3-D contractile cardiac grafts using gelatin sponges and synthetic biodegradable polymers. Leor and colleagues ${ }^{8}$ reported the formation of bioengineered cardiac grafts with 3-D alginate scaffolds. Other groups have studied the use of extracellular matrix (ECM) scaffolds. Eschenhagen and coworkers ${ }^{9}$ engineered 3-D heart tissue by gelling a mixture of cardiomyocytes and collagen. The engineered heart tissue survived and matured after implantation on uninjured hearts. ${ }^{10}$ Shimizu and colleagues ${ }^{11}$ have developed a novel approach of culturing cell sheets without scaffolds using a temperature-responsive polymer. Several cell sheets were layered on top of each other to create thicker grafts. ${ }^{12,13}$ These ECM scaffolds provide several options for obtaining functional cardiac tissue.

As an alternative approach, we have developed an in vitro system for creating sheets of cardiomyocytes on a mesh consisting of ultrafine fibers. ${ }^{14}$ Our device consists of a thin, highly porous, nonwoven fibrous mesh stretched across a wire ring. Ultrafine polymer nanofibers were produced by means of electrostatic fiber spinning and collected on wire rings in the form of a nonwoven mesh. Because of the small fiber diameters, the mesh has a high specific surface area and porosity that are beneficial for cell attachment and proliferation. ${ }^{15-17}$ In our previous study cardiomyocytes from neonatal Lewis rats were cultured on thin electrospun poly( $\epsilon$-caprolactone) meshes (PCL meshes). It was found that the meshes were sufficiently flexible and did not impede the myocyte contractions. Furthermore, this novel scaffold can be fabricated in specific shapes and is
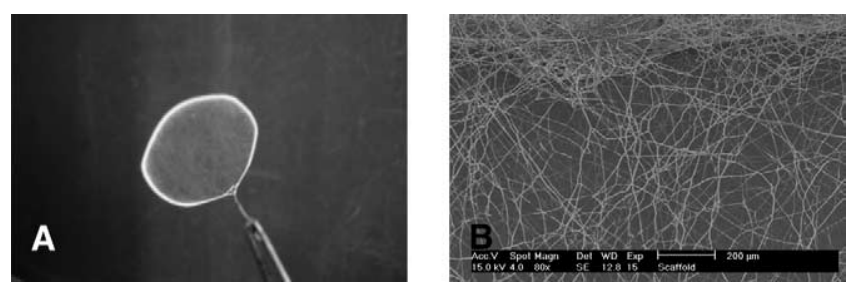

Figure 1. A, Gross view of single-layer scaffold. A thin, nonwoven fibrous mesh is suspended across a wire ring with a diameter of about $15 \mathrm{~mm}$. The thickness of the mesh is about $10 \mu \mathrm{m}$. The wire ring acts as a passive load to condition cardiomyocytes during in vitro culture. B, Scanning electron microscopic micrograph of scaffold. The electrospun fibers have an average diameter of $\mathbf{2 5 0}$ $\mathrm{nm}$. The pores are interconnected and are much larger than the fibers. The topography of the nonwoven mesh resembles that of an extracellular matrix and facilitates cell attachment.

easy to handle. Our results demonstrate that these cellpolymer constructs can be cultured over extended periods on thin PCL meshes in vitro. However, thicker grafts are required to obtain sufficient function. It is hypothesized that a clinically relevant cardiac graft will require a vasculature to provide sufficient perfusion of oxygenated blood. As an intermediate step toward a thick, vascularized cardiac graft, it is important to assess the ability to increasing the thickness without a vasculature and determine the maximum thickness before core ischemia is observed in the graft.

Here we report the extension of our concept and the development of a multilayer system as an intermediate step toward functional cardiac grafts. Individual grafts were stacked on top of each other, resulting in a multilayered graft. The layered grafts were cultured for 2 weeks in vitro and characterized by means of histology, immunohistochemistry, and scanning electron microscopy.

\section{Materials and Methods Scaffolds}

The detailed scaffold fabrication procedure is described elsewhere. ${ }^{14}$ Briefly, scaffolds were made by using the electrospinning process. A $10 \mathrm{wt}$ \% stock solution was prepared by dissolving PCL (molecular weight, $80 \mathrm{kDa}$; Aldrich, Milwaukee, Wis) in a 1:1 mixture of chloroform and methanol (Sigma, St Louis, Mo). The polymer solution was delivered at a constant flow rate $(0.1 \mathrm{~mL} /$ $\mathrm{min}$ ) to a metal tube (inner diameter, $1 \mathrm{~mm}$; Cole-Parmer, Vernon Hills, Ill) connected to a high-voltage power supply (Gamma High Voltage Research, Ormond Beach, Fla). On applying a voltage of $12 \mathrm{kV}$, the fiber-spinning process commenced, and solid fibers were collected in the form of nonwoven mesh on nickel-chrome wire rings (ring diameter, $15 \mathrm{~mm}$; wire thickness, $0.08 \mathrm{~mm}$; McMaster-Carr, Atlanta, Ga). A gross view of the scaffold is shown in Figure 1, $A$. The thickness of an individual scaffold is 10 $\mu \mathrm{m}$. The surface topography is shown in Figure $1, B$. The scaffold consists of a 3-D mesh of ultrafine fibers with an ECM-like 
topography. Typical fiber diameters range from $100 \mathrm{~nm}$ to $5 \mu \mathrm{m}$, although occasional outliers are observed. The average fiber diameter is $250 \mathrm{~nm}$. The scaffolds were stored in a dessicator. Before cell seeding, the scaffolds were placed in $70 \%$ ethanol overnight and then immersed in a purified collagen solution (Cohesion Technologies, Palo Alto, Calif).

\section{Cell Source and Seeding}

Primary cultures of cardiomyocytes were prepared according to previously published procedures. ${ }^{14}$ Ventricles obtained from 3-day-old Lewis rats (Charles River Laboratories, Wilmington, Mass) were minced into small segments $\left(<1 \mathrm{~mm}^{3}\right)$ and enzymatically digested for 10 minutes in a $37^{\circ} \mathrm{C}$ water bath with $0.25 \%$ trypsin (Gibco, Grand Island, NY) and $90 \mathrm{U} / \mathrm{mL}$ collagenase 2 (Worthington, Lakewood, NJ). The dissociated cells were plated on a Petri dish for 20 minutes at $37^{\circ} \mathrm{C}$ in culture medium. The culture medium consisted of 40\% M-199 medium (Gibco), 54\% Earle's Balanced Salt Solution (Sigma), and 6\% fetal bovine serum (Gibco) supplemented with $100 \mathrm{U} / \mathrm{mL}$ penicillin and streptomycin (Gibco). Nonadherent cells were removed and counted with a cell counter. The cells were seeded on the scaffolds at a concentration of 4 to 5 million cells per scaffold and incubated at $37^{\circ} \mathrm{C}$ in a humidified atmosphere with $5 \% \mathrm{CO}_{2}$. Cell viability was assessed daily by means of light microscopy and videomicroscopy. In addition, culture medium was exchanged daily for the duration of the experiment.

\section{Manipulation of Individual Grafts Into Multilayered Grafts}

After culturing for 5 to 7 days, layering of the individual grafts was performed. The grafts were gently placed on top of each other. The culture medium was gently removed and the layered constructs were incubated for 2 hours at $37^{\circ} \mathrm{C}$ to improve interlayer attachment. Culture medium was slowly added thereafter. In this study up to 5 grafts were stacked on top of each other. The multilayer grafts were cultured under the same conditions as the single-layer grafts for 14 days from harvest. Single-layer grafts were also cultured as controls.

\section{Histologic and Immunohistochemical Examinations}

The cross-sections of the grafts were analyzed after 14 days of in vitro culture. After fixation with $4 \%$ paraformaldehyde, the grafts were embedded in paraffin. Plane sections and cross-sections (5 $\mu \mathrm{m})$ were cut and stained with hematoxylin and eosin (H\&E) for general assessment. For cell F-actin staining, the grafts were stained with a 1:20 dilution of rhodamine-conjugated phalloidin (Sigma) for 2 hours and counterstained with $2 \mu \mathrm{g} / \mathrm{mL} 4^{\prime}, 6^{\prime}$ diamidino-2-phenylindole hydrochloride (DAPI; Sigma) for 2 minutes. Masson trichrome staining (Sigma) was also performed for collagen assessment.

Immunohistochemical staining for cardiac troponin I was performed on graft cross-sections. Sections were incubated overnight with a 1:100 dilution of anti-cardiac troponin I antibody (Spectral Diagnostics, Whitestone, Va), followed by a 1:200 dilution of biotin-conjugated anti-mouse $\mathrm{IgG}$ antibody (DAKO, Glostrup, Denmark) for 2 hours. The specimens were then incubated with avidin-biotin complex reagent and 3,3' -diaminobenzidine (Sigma). Staining for type I and II collagen was performed in an analogous manner to the troponin staining by using anti-collagen antibodies (Accurate Chemical \& Scientific Corporation, Westbury, NY). To examine the presence of gap junctions, frozen sections were prepared after acetone fixation, followed by incubation with a 1:100 dilution of anti-connexin43 antibody (Chemicon, Temecula, Calif) overnight. Then specimens were incubated with a 1:200 diluted fluorescein isothiocyanate-labeled anti-mouse IgG antibody. Images were obtained with a digital camera in a Nikon fluorescence microscope.

\section{Scanning Electron Microscopy}

The fixed grafts were rinsed in $0.1 \mathrm{~mol} / \mathrm{L}$ phosphate-buffered saline (Gibco) and dehydrated in increasing concentrations of ethanol. Dehydrated grafts were immersed in hexamethyldisilazane (Fluka Chemical Corp, Milwaukee, Wis) for 15 minutes and dried overnight in a dessicator. After drying, the samples were mounted on aluminum stubs, sputter coated with gold-palladium, and viewed in a scanning electron microscope (Philips XL-20; FEI, Hillsboro, Ore) with an accelerating voltage of 10 to $20 \mathrm{kV}$.

\section{Results}

The neonatal rat cardiomyocytes attached well on the electrospun PCL scaffolds. On day 3, the cardiomyocytes started to contract. Between days 5 and 7 , individual grafts were layered on top of each other and cultured as a multilayered graft until day 14. Initially, weak and unsynchronized contractions were observed, but as time progressed, the contractions became stronger and more synchronized. Figure 2, $A$ and $B$, shows a comparison of the cross-sections of a monolayer and 5-layer graft, respectively. H\&E staining revealed that the individual layers are connected. Furthermore, stratified cardiac tissue between connective tissue was observed as indicated by means of Masson trichrome staining. This is shown in Figure 2, $C$ and $D$. Figure 3 shows a planar view of the 5-layered graft by means of $H \& E$ and Masson trichrome staining, respectively.

Sections were stained with cardiac-specific troponin I to verify the cell phenotype. As Figure 4, A, shows, cells in the graft stain positively for cardiac troponin I. Several cardiac layers are shown to adhere intimately and appear to have become homogeneous tissue. Staining for collagen demonstrated that the ECM consisted mainly of collagen type I (Figure 4, B). The scaffold is difficult to detect because of the small fiber diameters. Immunofluorescent images with anti-connexin43 antibody demonstrated diffuse formation of gap junctions in the layered grafts. The nuclei were counterstained with DAPI (Sigma; Figure 4,C).

Scanning electron microscopic analysis of the surface shows an abundant coverage with several cell layers (Figure $5, A)$. A cross-sectional view reveals that cells have penetrated the entire thickness of the scaffold, although the cell density is slightly higher on the surfaces. After 2 weeks, nondegraded PCL fibers remain, and these can also be seen (Figure 5, B). 
A

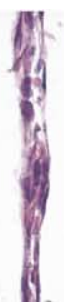

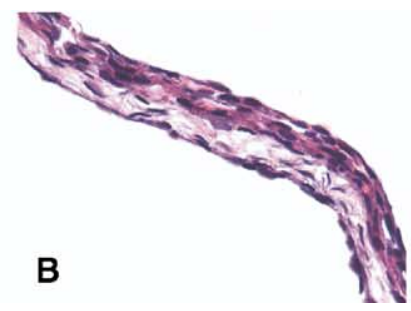

C

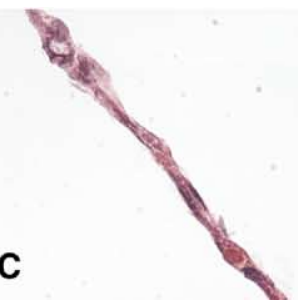

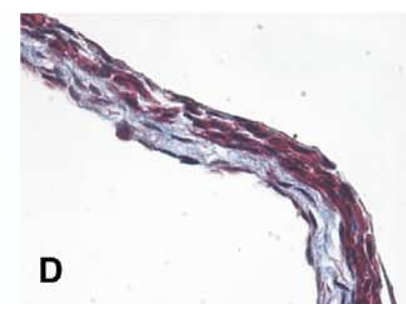

Figure 2. A, Cross-sectional view of a single graft. Hematoxylin and eosin staining shows that cells have attached to the scaffold and proliferate well through the entire thickness of the scaffold. (Original magnification $400 \times$.) $B$, Cross-sectional view of a 5-layer graft. Cardiomyocytes were first cultured on individual scaffolds to induce cell attachment. After 5 to 7 days, 5 scaffolds were gently placed on top of each other and maintained in culture. The 5-layer construct has the appearance of a single tissue segment, as shown here with hematoxylin and eosin staining. There is no evidence of physical gaps, and it appears the individual layers have fused well. (Original magnification $400 \times$.) C, Cross-sectional view of a single graft. Masson trichrome staining reveals the presence of muscle and collagen throughout the entire specimen. (Original magnification $400 \times$.) D, Cross-sectional view of a 5-layer graft. Masson trichrome staining reveals the presence of collagen. In addition, a stratified cardiac muscle-like structure can be seen. (Original magnification $400 \times$.)
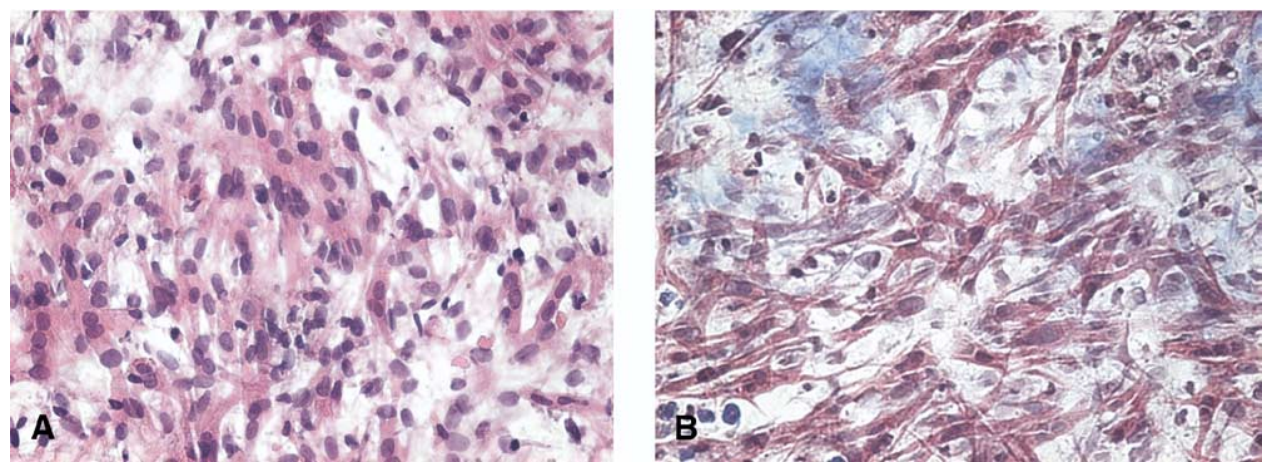

Figure 3. A, Surface view of a 5-layer graft visualized with hematoxylin and eosin staining. Cells are spread across the entire surface. The appearance of overlapping nuclei is attributed to cells adhering to pores in the scaffold below the surface. (Original magnification $200 \times$.) B, Surface view of a 5-layer graft visualized with Masson trichrome staining. Cardiac muscle-like structures can be seen. (Original magnification $200 \times$.)

A

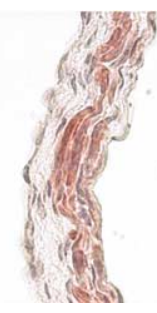

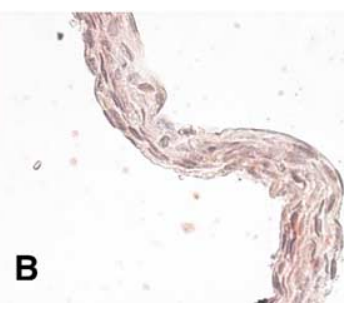

Figure 4. A, Immunohistochemical staining for cardiac troponin I in a 5-layered graft. Cardiac troponin I-positive cells are seen throughout the entire cross-section of the graft. (Original magnification $400 \times$.) B, Immunohistochemical staining for collagen type $I$ in a 5-layered graft. Collagen is abundantly present throughout the entire specimen. (Original magnification $400 \times$.) C, Immunohistochemical staining for connexin43 in a 5-layered graft. Diffuse gap junctions between the cells on 5 -layered grafts can be seen. The nuclei have been counterstained with $4^{\prime}, 6^{\prime}$-diamidino-2-phenylindole hydrochloride. (Original magnification $400 \times$.) 

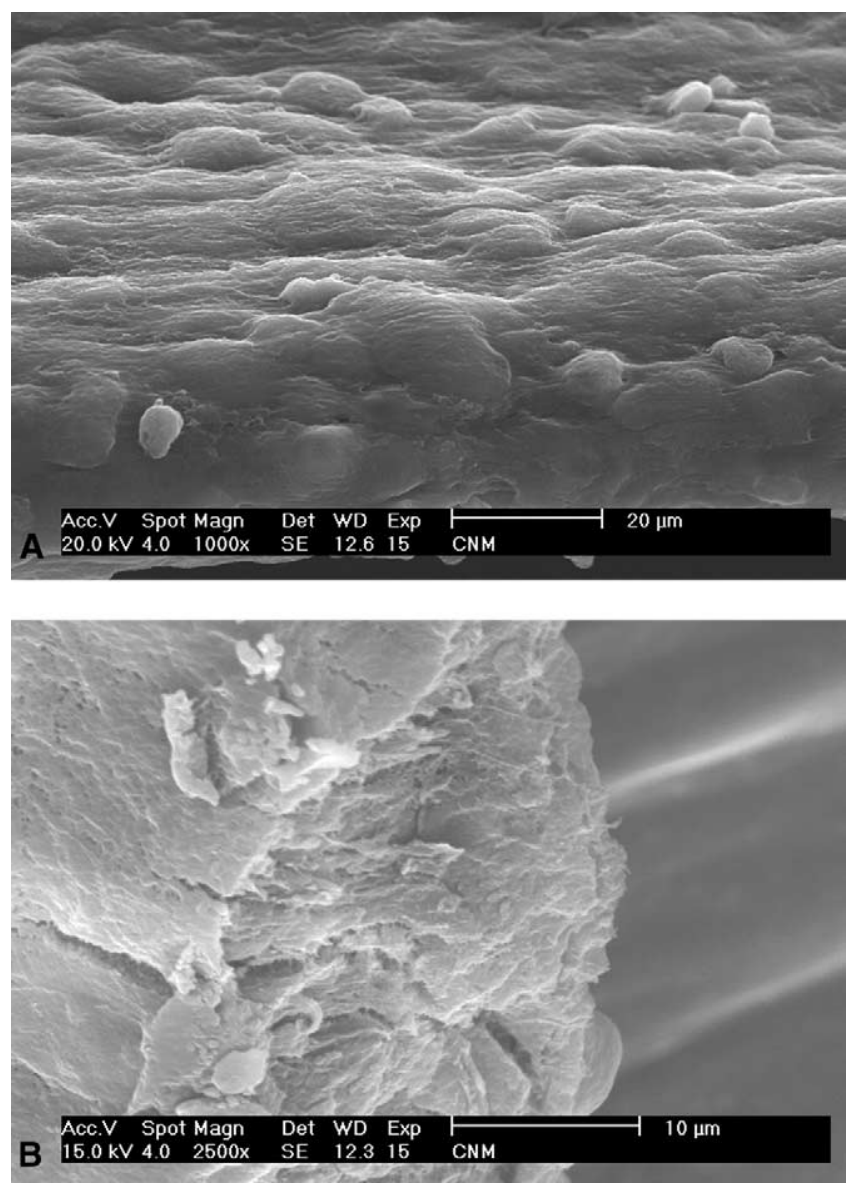

Figure 5. A, Scanning electron microscopic micrograph of a 5-layer graft. The surface is densely covered with cells after 2 weeks of culture. Scale bar $=20 \mu \mathrm{m}$. B, Scanning electron microscopic micrograph of a 5-layer graft. The cells have penetrated the entire cross-section of the specimen. Nondegraded fibers of the scaffold are also present. Scale bar $=10 \mu \mathrm{m}$.

\section{Discussion}

Primary neonatal rat cardiomyocytes were cultured on novel polymer meshes and successfully layered into multilayer grafts. The individual layers formed homogenous tissue, and cardiac tissue-like structures, such as sarcomeres and gap junctions, were observed. Five-layer grafts contracted macroscopically in a synchronized manner in vitro during the entire experimental period.

One key requirement for clinically relevant cardiac grafts is sufficient function immediately after implantation. This requirement translates into a need for a high concentration of cardiac muscle cells. This report outlines a novel approach toward myocardial grafts. The approach taken here is based on our recently developed concept. An individual graft approximates a monolayered cardiac muscle cell sheet and can be used as an independent system for cardiac cell culture. However, because of its limited thickness $(<10$ $\mu \mathrm{m})$, it appears unlikely that it will provide sufficient function when used alone to cover an infarcted area. Here we demonstrate the ability of these grafts to form a building block for clinically relevant cardiac grafts by forming multilayered structures. The grafts can be stacked to obtain thicker grafts, and they fuse well with each other. The presence of connexin43 indicates that the cells are rebuilding gap junctions, and synchronized contractions in multilayered grafts are observed. The construct thickness can be regulated by the number of layers. The use of the scaffold also allows control over the 2-dimensional shape and size. Because of its ECM-like topography, the nanofibrous mesh provides sufficient surface area for cell attachment. Because the fiber diameters are at least one order of magnitude smaller than the cells, the number of cell-cell contacts is maximized, thereby allowing the formation of electrical and morphologic connections. Implant studies are currently underway to further assess the clinical potential of our multilayered cardiac graft system.

However, because cardiac muscle cells have high metabolic requirements, it is anticipated that a cardiac graft with clinically relevant dimensions will also require a feeder vasculature to provide sufficient nutrients and oxygen. Previous cardiac tissue-engineering studies have noted that the cell density is highest around the periphery of the grafts, whereas the interior had either necrotic or no cells because of insufficient oxygen perfusion if the thickness of the graft exceeded $100 \mu \mathrm{m} .{ }^{18}$ To this end, experiments are currently under way to combine our layered approach with endothelial cell layers to arrive at a thick graft. Another approach is to incorporate a preformed vasculature that is currently under development in out laboratory. ${ }^{19}$ A simple network of endothelialized channels with an inlet and outlet would also be of interest for the clinician because the inlet and outlet could be anastomosed to the native coronary vessels of the recipient heart.

Cell sourcing remains a critical issue for cell transplantation. Because cardiomyocytes are terminally differentiated cells, they do not divide significantly, and it is difficult to obtain autologous cardiomyocytes for transplantation. Various cell types have recently been assessed for transplantation into myocardial tissues, including myoblasts and multipotent endothelial cells. ${ }^{20,21}$ Furthermore, stem cell studies indicate the possibility of obtaining cardiomyocytes from various stem cells. ${ }^{22}$ Autologous cells, in particular multipotent cells derived from the bone marrow, might be a desirable cell source. Important pioneering tissue-engineering studies have recently been reported by Shin'oka's group. ${ }^{23-25}$

In conclusion, electrospun scaffolds have been shown to be very useful for fabricating 3-D cardiac grafts in vitro. Although the current scaffolds provide an ECM-like topography, aspects of the unique geometry and sheet-like microstructure of the 
heart (eg, anisotropy and asymmetry) have not been addressed yet. Our experiments (data not shown) indicate that electrospun nanofibers can be aligned to improve their orientation, but the effect on cell organization remains to be seen. Although clinical applications of cardiac tissue engineering are still far away, autologous cardiac grafts have potential as an in vitro heart tissue model and, depending on progress in the development of new techniques to create blood vessels and the discovery of an appropriate cell source, as improved cardiac graft materials in clinical tissue repair.

\section{References}

1. Kirklin JK, Pambukian SV, McGiffin DC, Benza RL. Current outcomes following heart transplantation. Semin Thorac Cardiovasc Surg. 2000;16:395-403.

2. Langer R, Vacanti JP. Tissue engineering. Science. 1993;260:920-6.

3. Shinoka T. Tissue engineered heart valves: autologous cell seeding on biodegradable polymer scaffold. Artif Organs. 2002;26:402-6.

4. Schmedlen RH, Elbjeirami WM, Gobin AS, West JL. Tissue engineered small-diameter vascular grafts. Clin Plast Surg. 2003;30: 507-17.

5. Li RK, Jia ZQ, Weisel RD, Mickle DA, Choi A, Yau TM. Survival and function of bioengineered cardiac grafts. Circulation. 1999;100(suppl II):II63-9.

6. Sakai T, Li RK, Weisel RD, Mickle DA, Kim ET, Jia ZQ, et al. The fate of a tissue-engineered cardiac graft in the right ventricular outflow tract of the rat. J Thorac Cardiovasc Surg. 2001;121:932-42.

7. Ozawa T, Mickle DA, Weisel RD, Koyama N, Ozawa S, Li RK. Optimal biomaterial for creation of autologous cardiac grafts. Circulation. 2002;106(suppl 1):I176-82.

8. Leor J, Aboulafia-Etzion S, Dar A, Shapiro L, Barbash IM, Battler A, et al. Bioengineered cardiac grafts: a new approach to repair the infarcted myocardium? Circulation. 2000;102(suppl 3):III56-61.

9. Eschenhagen T, Fink C, Remmers U, Scholz H, Wattchow J, Weil J, et al. Three-dimensional reconstitution of embryonic cardiomyocytes in a collagen matrix: a new heart muscle model system. FASEB J. 1997;11:683-94.

10. Zimmermann WH, Didie M, Wasmeier GH, Nixdorff U, Hess A, Melnychenko I, et al. Cardiac grafting of engineered heart tissue in syngenic rats. Circulation. 2002;106(suppl 1):I151-7.

11. Shimizu T, Yamato M, Kikuchi A, Okano T. Two-dimensional manipulation of cardiac myocyte sheets utilizing temperature-responsive culture dishes augments the pulsatile amplitude. Tissue Eng. 2001;7:141-51.
12. Shimizu T, Yamato M, Isoi Y, Akutsu T, Setomaru T, Abe K, et al. Fabrication of pulsatile cardiac tissue grafts using a novel 3dimensional cell sheet manipulation technique and temperature responsive cell culture surfaces. Circ Res. 2002;90:e40.

13. Shimizu T, Yamato M, Kikuchi A, Okano T. Cell sheet engineering for myocardial tissue reconstruction. Biomaterials. 2002;24:2309-16.

14. Shin M, Ishii O, Sueda T, Vacanti JP. Contractile cardiac grafts using a novel nanofibrous mesh. Biomaterials. 2004;25:3717-23.

15. Huang L, Nagapudi K, Apkarian RP, Chaikof EL. Engineered collagen-PEO nanofibers and fabrics. J Biomater Sci Polym Ed. 2001; 12:979-93.

16. el-Kenawy R, Bowlin GL, Mansfield K, Layman J, Simpson DG, Sanders EH, et al. Release of tetracycline hydrochloride from electrospun poly(ethylene-co-vinylacetate), poly(lactic acid), and a blend. $J$ Control Release. 2002;81:57-64.

17. Yoshimoto H, Shin YM, Terai H, Vacanti JP. A biodegradable nanofiber scaffold by electrospinning and its potential for bone tissue engineering. Biomaterials. 2003;24:2077-82.

18. Bursac N, Papadaki M, Cohen RJ, Schoen FJ, Eisenberg SR, Carrier RL, et al. Cardiac muscle tissue engineering: toward an in vitro model for electrophysiological studies. Am J Physiol Heart Circ Physiol. 1999;277:H433-44.

19. Shin M, Matsuda K, Ishii O, Terai H, Kaazempur-Mofrad M, Detmar $\mathrm{M}$, et al. Endothelialized vascular networks in microfabricated poly(dimethyl siloxane). Biomed Microdevices. 2004;6:269-78.

20. Murry CE, Wiseman RW, Schwartz SM, Hauschka SD. Skeletal myoblast transplantation for repair of myocardial necrosis. J Clin Invest. 1996;98:2512-23.

21. Condorelli G, Borello U, De Angelis L, Latronico M, Sirabella D, Coletta M, et al. Cardiomyocytes induce endothelial cells to transdifferentiate into cardiac muscle: implications for myocardium regeneration. Proc Natl Acad Sci U S A. 2001;98:10733-8.

22. Kehat I, Kenyagin-Karsenti D, Snir M, Segov H, Amit M, Gepstein A, et al. Human embryonic stem cells can differentiate into myocytes with structural and functional properties of cardiomyocytes. J Clin Invest. 2001;108:407-14

23. Shin'oka T, Imai Y, Ikada Y. Transplantation of a tissue-engineered pulmonary artery. $N$ Engl J Med. 2001;344:532.

24. Matsumura G, Miyagawa-Tomita S, Shin'oka T, Ikada Y, Kurosawa $\mathrm{H}$. First evidence that bone marrow cells contribute to the construction of tissue-engineered vascular autografts in vivo. Circulation. 2003; 108:1729-34.

25. Naito Y, Imai Y, Shin'oka T, Kashiwagi J, Aoki M, Watanabe M, et al. Successful clinical application of tissue-engineered graft for extracardiac Fontan operation. J Thorac Cardiovasc Surg. 2003; $125: 419-20$ 